\title{
Meta
}

Journal des traducteurs

Translators' Journal

\section{Une didactique de la traduction à partir des langues périphériques : le " régime spécial " à l'ÉSIT}

\section{Geneviève Roux-Faucard}

Volume 50, numéro 1, mars 2005

Enseignement de la traduction dans le monde

Teaching Translation Throughout the World

URI : https://id.erudit.org/iderudit/010668ar

DOI : https://doi.org/10.7202/010668ar

Aller au sommaire du numéro

Éditeur(s)

Les Presses de l'Université de Montréal

ISSN

0026-0452 (imprimé)

1492-1421 (numérique)

Découvrir la revue

Citer cet article

Roux-Faucard, G. (2005). Une didactique de la traduction à partir des langues périphériques : le « régime spécial » à l’ÉSIT. Meta, 50(1), 194-209.

https://doi.org/10.7202/010668ar
Résumé de l'article

La mondialisation favorise le développement des écoles de traduction, mais elle est accompagnée par un certain type de relation entre les langues et les cultures : les langues les plus parlées sont favorisées au détriment des langues périphériques et des cultures qu'elles transmettent. Pour freiner cette évolution, l’ÉSIT forme des locuteurs de langues de petite diffusion, destinés à devenir traducteurs « en B » et à faire connaître leur culture au reste du monde ; les formateurs sont des enseignants qui n'ont pas accès à la langue maternelle des étudiants. Sur la base d'exemples concrets, cet article montre la spécificité de cet enseignement, qui repose sur les principes de la Théorie Interprétative de la Traduction et en constitue une illustration convaincante.
Ce document est protégé par la loi sur le droit d'auteur. L'utilisation des services d’Érudit (y compris la reproduction) est assujettie à sa politique d'utilisation que vous pouvez consulter en ligne.

https://apropos.erudit.org/fr/usagers/politique-dutilisation/ 


\title{
Une didactique de la traduction à partir des langues périphériques: le «régime spécial » à l'ÉSIT
}

\author{
GENEVIÈVE ROUX-FAUCARD \\ Université Sorbonne Nouvelle (Paris III), Paris, France \\ genevieve.roux@free.fr
}

\begin{abstract}
RÉSUMÉ
La mondialisation favorise le développement des écoles de traduction, mais elle est accompagnée par un certain type de relation entre les langues et les cultures: les langues les plus parlées sont favorisées au détriment des langues périphériques et des cultures qu'elles transmettent. Pour freiner cette évolution, l'ÉSIT forme des locuteurs de langues de petite diffusion, destinés à devenir traducteurs «en $B$ » et à faire connaître leur culture au reste du monde; les formateurs sont des enseignants qui n'ont pas accès à la langue maternelle des étudiants. Sur la base d'exemples concrets, cet article montre la spécificité de cet enseignement, qui repose sur les principes de la Théorie Interprétative de la Traduction et en constitue une illustration convaincante.
\end{abstract}

\begin{abstract}
Globalization promotes the development of translation schools, but it is accompanied by an alarming phenomenon: major languages will be increasinlgy encouraged to the detriment of languages of lesser importance and the cultures they convey. In order to slow down the process, ÉSIT teaches speakers of minor languages to translate into a better known language and thus make their culture known to the rest of the world. The teachers do not need to be familiar with the students' mother tongue. This article uses practical examples to illustrate the strategies that make this approach possible. It is a convincing illustration of the principles behind the "theory of meaning" (Théorie Interprétative de la Traduction) on which it is based.
\end{abstract}

\section{MOTS-CLÉS/KEYWORDS}

enseignement de la traduction, langues de faible diffusion, communication interculturelle, mondialisation, traduction en $\mathrm{B}$

Les écoles de traduction forment des professionnels. Le succès de celles-ci sur le marché de la formation dépend de la réussite de ceux-là sur le marché du travail, de leur adaptation à la demande et aux exigences de l'économie. Soit. La mondialisation, qui s'accompagne, entre toutes les régions du globe, d'un accroissement des échanges d'information et des besoins de concertation, offre actuellement une conjoncture favorable à ces acteurs de la communication interculturelle que sont les traducteurs et les interprètes et, par conséquent, aux instituts qui les forment. Sans doute. Faut-il pour autant accepter que les écoles de traduction se contentent de jouer le jeu du marché, sans jeter un regard critique sur les évolutions probables? Car la soumission inconditionnelle aux lois de l'économie, si elle garantit le succès à court terme, n'en comporte pas moins, à plus long terme, des risques majeurs, dont 
les langues et les cultures pourraient bien faire les frais, ainsi que, par voie de conséquence, les traducteurs et leurs instituts de formation.

Dans ce contexte, nous nous proposons d'évoquer un aspect original de l'enseignement de la traduction: la formation de traducteurs ayant comme langue maternelle une langue de petite diffusion, pilotée par des enseignants ne connaissant pas cette langue. Sous le nom de «régime spécial», ce type de formation est dispensé depuis de nombreuses années à l'ÉSIT ${ }^{1}$; il en constitue une spécificité et y est l'objet d'une sollicitude toute particulière. Nous montrerons que, loin d'être une pratique marginale justiciable d'incrédulité et de scepticisme, cet enseignement offre, par ses objectifs, une réponse adaptée à une situation mondiale préoccupante; puis, en nous basant sur des situations concrètes, nous montrerons qu'il repose sur une didactique fortement étayée par la Théorie Interprétative de la Traduction, qui constitue son assise théorique et dont il offre une illustration particulièrement convaincante.

\section{Les risques de la mondialisation pour les langues et les cultures}

La mondialisation telle que nous la vivons, c'est-à-dire dominée par le poids du monde anglo-saxon, est accompagnée par un certain type de structure relationnelle entre les différentes langues. Pour évoquer rapidement ce nouvel état linguistique du monde et les évolutions qu'il induit, je m'appuierai sur le modèle «gravitationnel » proposé par L.-J. Calvet ${ }^{2}$. De même que les corps célestes ont, les uns par rapport aux autres, une position et un mouvement qui sont déterminés par leur masse, de la même façon les langues constituent, à chaque époque, un système où la position d'une langue par rapport aux autres est déterminée par sa "valeur», donnée intégrant son nombre de locuteurs primaires et secondaires, ainsi que d'autres facteurs, économiques et politiques, que l'on regroupera sous le terme de «prestige». Pour notre époque, Calvet distingue quatre niveaux dans cette "vaste galaxie», l'anglais occupant la position la plus centrale. Les langues de second niveau, qui gravitent autour de lui, sont au nombre d'une dizaine (dont le russe, l'arabe, le chinois, le français, l'espagnol). Autour de chacune d'elles gravitent des langues de troisième niveau: cent à deux cents, dont le japonais, l'allemand et le bambara. Toutes les autres langues, au nombre de quatre à cinq mille, réparties autour des "planètes» de troisième niveau, sont dites périphériques ${ }^{3}$ (la plupart des langues de l'Afrique, langues minoritaires de l'Europe et de l'Asie, langues "premières» d'Amérique du Nord et du Sud, etc.). Cette image de «constellations linguistiques», qui n'est qu'une modélisation ${ }^{4}$, permet d'appréhender certains mécanismes du système et d'illustrer sa dynamique interne.

Quand un individu choisit d'acquérir une langue étrangère - ce choix reposant «d'abord sur la recherche de l'efficacité»-, il choisit généralement une langue du niveau immédiatement supérieur: au Mali (d'après L.-J. Calvet), un locuteur du peul apprend le bambara, un locuteur du bambara apprend le français. Comme partout ailleurs, un locuteur français apprend l'anglais. Quant aux anglophones, on sait bien qu'ils "présentent une tendance marquée au monolinguisme». Cette analyse correspond bien à ce que nous constatons en observant le «marché» des langues dans les pays qui nous sont proches. Il existe une "attraction», "tendance au bilinguisme orienté vers le centre», force que nous appellerons donc centripète, qui contribue à accroître le prestige des langues les plus centrales, et surtout celui de la langue «hypercentrale», par rapport aux langues périphériques. 
En restant dans la logique très éclairante de ce modèle, ajoutons quelques remarques concernant plus spécifiquement la traduction. Parmi les sujets ayant acquis une bonne maîtrise d'une langue étrangère, ceux qui deviendront traducteurs traduiront majoritairement de la langue acquise vers leur langue maternelle, donc (sauf dans le cas de traducteurs anglophones) d'une langue plus centrale vers une langue plus périphérique. De plus, le prestige des cultures portées par les langues plus centrales induit, aux niveaux plus périphériques, une demande d'information et de consommation culturelle, alors que la culture la plus centrale se préoccupe, comme on le sait, assez peu des «mondes» qui l'entourent. Ainsi le flux des traductions est-il, par le fonctionnement même de l'offre comme par celui de la demande, orienté du centre vers la périphérie. En assurant la diffusion des cultures plus centrales, ce flux centrifuge contribue, lui aussi, à accroître leur prestige. Mais cet effet automatique peut, en outre, se trouver renforcé par une stratégie mise en œuvre par les États qui représentent ces cultures: en assurant la promotion de ces dernières, ils engrangent en effet des bénéfices économiques directs (commercialisation de produits culturels) et indirects (exportation de biens de consommation) considérables; quant à leur poids politique, sa conservation et son renforcement passent par la mise en circulation, elle aussi tributaire de la traduction, de notions, d'informations et de choix idéologiques.

Cette description peut être quotidiennement vérifiée dans tous les domaines, du technique au littéraire ${ }^{5}$, et à tous les niveaux du système. Une étude réalisée à l'occasion de la dernière Foire du livre à Francfort a établi que $50 \%$ des traductions publiées (tous pays compris) se font à partir de l'anglais; le flux inverse (œuvres traduites, toutes langues confondues, entrant dans le système éditorial américain) ne compte que pour $3 \%$, la majorité de ces traductions étant faites à partir des langues de niveau immédiatement inférieur (le français, suivi de l'allemand et de l'espagnol) ${ }^{6}$. La communication culturelle qui s'établit par l'intermédiaire des traductions est donc, effectivement, massivement centrifuge: c'est un transfert et non un échange, les cultures plus périphériques étant destinées à accueillir des éléments exogènes en provenance des cultures plus centrales, à recevoir les cultures dominantes et, à terme, à être modifiées par elles. Le rôle réservé à la traduction dans le fonctionnement de la nouvelle "galaxie des langues » est donc tel qu'elle ne travaille pas forcément dans le sens d'une véritable circulation des idées, qu'elle n'est pas automatiquement un dispositif d'échange et de négociation, mais peut être instrumentalisée au service d'un dessein hégémonique.

Le système constitué par les langues et les cultures du monde, on le voit, n'est pas immobile; les deux mouvements repérés en son sein, mouvement centripète de l'apprentissage des langues étrangères et mouvement centrifuge des flux traductionnels, contribuent, l'un et l'autre, à augmenter la «masse» gravitationnelle de la langue centrale, de la culture qu'elle véhicule et des États qui les représentent. Ce fonctionnement menace l'existence même de la diversité. Concernant les langues et les cultures périphériques, on sait que cette évolution est déjà largement entamée ${ }^{7}$. À moyen terme, on peut penser que les langues et les cultures « de troisième niveau » peuvent, elles aussi, être menacées d'érosion. Ce déséquilibre croissant priverait l'humanité de l'enrichissement et de la régulation résultant du dialogue culturel et de la multipolarité. À l'horizon, on entrevoit l'image d'un monde culturellement appauvri ${ }^{8}$ qui aurait de moins en moins besoin de communication interculturelle - et de traduction. 


\section{Quelle réponse les instituts de traduction peuvent-ils donner?}

Une réponse répandue à tous les niveaux de décision est celle appelée par Calvet «planification par défaut, qui consiste à ne rien faire, ou à laisser faire». On peut penser que les responsables des instituts de traduction ne sauraient, pas plus que d'autres, intervenir dans le fonctionnement du système, ni s'opposer à son instrumentalisation, ni infléchir les évolutions probables. Cependant, le rôle d'accélérateur joué par le mécanisme du choix dans l'apprentissage d'une langue étrangère et par celui des flux traductionnels interdit que l'on s'en tienne à ce simple constat d'impuissance justifiant l'inaction. Si l'on estime souhaitable de freiner l'érosion de la diversité culturelle et de défendre un monde multipolaire, il convient de tenter, sur le terrain qui est le nôtre, de maîtriser le fonctionnement de ces mécanismes et de leur imposer une régulation "équitable ${ }^{9}$. Autrement dit, de travailler contre cette «pesanteur» que le modèle gravitationnel nous a permis de voir à l'œuvre.

D'une façon assez générale, «travailler contre la pesanteur» peut prendre la forme de plusieurs objectifs. Sur le plan strictement linguistique, il s'agit d'éviter que, dans les domaines de la vie économique, diplomatique, politique, etc., tous les échanges, qu'ils soient écrits ou oraux, ne passent par l'anglais, ou, pire encore, que tout ne se passe en anglais. Cela exige que la communication entre locuteurs de langue de second ou de troisième niveau (communication «horizontale») soit possible sans passer par l'intermédiaire d'une langue plus centrale ${ }^{10}$. De plus, les pays ayant une langue de second ou de troisième niveau doivent faire en sorte de pouvoir communiquer dans leur langue avec les pays de langue périphérique, pour éviter que ceux-ci ne soient tributaires de la seule langue centrale et contraints de passer par son relais dans les échanges internationaux.

Sur le plan culturel, «travailler contre la pesanteur» signifie faire en sorte que les cultures plus périphériques ne soient pas seulement destinées à être réceptrices, mais aient la possibilité d'être reçues par les cultures plus centrales. Au lieu que leur patrimoine, faute d'un canal de transmission, disparaisse dans l'ignorance générale de l'opinion publique mondiale, puis, du fait de l'extinction de leur langue propre, se perde sur les lieux mêmes de ces cultures, cela signifie accueillir ce patrimoine culturel, sous ses formes d'expression actuelles (industrie cinématographique, production musicale, édition), à l'extérieur de son territoire et lui donner ainsi une chance d'être préservé et de se développer. Pour les cultures plus centrales, cet accueil est une chance d'ouverture et d'enrichissement.

Ces objectifs sont tels que tous les acteurs de la vie internationale, particulièrement dans les domaines de l'économie et des marchés financiers, ne sont pas également disposés à les adopter; il est certain que, en termes de stricte efficacité à court terme, le rapport entre coût et rentabilité ne parle pas en faveur d'une politique linguistique et culturelle équitable. Mais on ne peut nier que des actions soient déjà engagées dans ce sens, que de nombreuses voix se fassent entendre en faveur de ces objectifs, comme on a pu le constater lors des débats suscités par la $32^{\mathrm{e}}$ session de la Conférence générale de l'Unesco à Paris (octobre 2003), et qu'elles soient soutenues par une large demande de l'opinion publique mondiale.

Mais cette demande restera inopérante si les moyens de la satisfaire ne sont pas créés. C'est ici qu'arrivent en première ligne les traducteurs, les interprètes et leurs instituts de formation. En effet, la mise en œuvre des objectifs généraux définis plus 
haut exige l'existence, sur le marché de l'emploi, de certains types de professionnels de la communication que les écoles de traduction sont les seules à pouvoir former. La communication «horizontale» suppose des traducteurs dans de très nombreuses combinaisons linguistiques comprenant des langues de second et de troisième niveau (donc des combinaisons sans anglais). Assurer la conservation des langues périphériques suppose aussi l'existence de traducteurs dans des combinaisons comportant l'une de ces langues et une langue plus centrale (dont des langues de second ou de troisième niveau). Dans ces types de combinaisons, il est besoin, enfin, de traducteurs capables de travailler vers la langue périphérique, mais surtout à partir d'elle. Or, les mécanismes de choix d'une langue étrangère font qu'il n'est guère possible de compter, pour les années à venir, sur un nombre suffisant de locuteurs des grandes langues occidentales ayant acquis un bon niveau dans l'une ou l'autre des langues périphériques et se trouvant en mesure de la traduire «vers le A». Ces futurs professionnels doivent donc être recrutés parmi des locuteurs de la langue périphérique, ayant une bonne maîtrise de l'autre langue et ayant acquis un niveau méthodologique suffisant pour traduire leur propre culture vers les cultures plus centrales, donc «vers le B».

Mettre sur le marché de la communication interculturelle des acteurs répondant à ces exigences, telle est, nous semble-t-il, la responsabilité actuelle des écoles de traduction face aux risques que la mondialisation fait peser sur les langues et les cultures. Restreindre les choix de formation à un petit nombre de combinaisons comportant obligatoirement l'anglais reviendrait à servir et à accélérer les évolutions actuelles. Défendre la diversité des langues et des cultures, au contraire, est possible en répondant par une offre à une demande perceptible de toutes parts: celle de professionnels diplômés et compétents prêts à s'engager dans les domaines les plus sensibles de la communication interculturelle.

\section{Le « régime spécial» à l'ÉSIT}

Dans ce qui suit, nous reprendrons le cas particulier de la formation de traducteurs ayant comme langue maternelle une langue périphérique. Dispenser une telle formation dans le cadre d'un institut européen ${ }^{11}$ pose des problèmes assez différents de l'enseignement «classique». L'ÉSIT bénéficie dans ce domaine d'une tradition déjà longue, puisque, dès les années soixante-dix, à l'initiative de Seleskovitch, la décision a été prise d'accueillir en filière «interprétation» des étudiants non européens, souvent d'origine asiatique, et dont la langue maternelle ne figurait pas au programme des cours réguliers de l'École. Plus tard, l'expérience s'est élargie à la filière «traduction », où elle s'est poursuivie et institutionnalisée. Cette expérience déjà ancienne lui permet actuellement de dispenser, sous le nom de «régime spécial», une formation qui a fait ses preuves ${ }^{12}$.

Sélectionnés parmi des postulants ayant effectué quatre ans d'études de français dans leur pays d'origine, les futurs traducteurs sont soumis pendant un an à une préparation intensive, aboutissant à l'obtention d'un «Certificat de méthodologie de la traduction». Ce cursus comprend, d'une part, des cours de méthodologie communs avec les étudiants du régime «classique» en année 1 ou 2 : linguistique, lecture active, terminologie, recherche documentaire, économie. Il comprend, d'autre part, des cours de traduction dans la combinaison linguistique propre des étudiants, rassemblés 
pour la circonstance en groupes de même langue maternelle. Parmi les langues régulièrement représentées dans ce programme, mentionnons le coréen, le japonais, le thaï, ainsi que quelques langues d'Europe centrale et orientale.

Cet entraînement, qui se fait exclusivement depuis la langue maternelle des étudiants (leur langue A) vers le français (leur langue B), doit pouvoir être effectué par des enseignants qui sont des spécialistes formés à la méthodologie de l'École et des chercheurs. En l'absence de formateurs ayant ces qualifications et ayant, en plus, l'une des langues concernées parmi leur langues de travail, il est piloté par des enseignants francophones, qui ne connaissent pas la langue maternelle des étudiants. Ces conditions de formation ont nécessité l'élaboration d'une didactique d'un type particulier, dont la suite de ce texte illustrera quelques aspects ${ }^{13}$, à l'aide d'exemples empruntés au travail effectué avec un groupe coréen ${ }^{14}$.

\section{Une séquence d'entraînement type}

Dans l'entraînement en «régime spécial», la plupart du travail se fait, comme en régime classique, sur des textes pragmatiques, car c'est à ce genre de documents que les jeunes traducteurs seront confrontés dans la majorité des cas. La recherche documentaire en domaine de spécialité faisant l'objet d'un autre module, c'est à la presse généraliste que les textes sont empruntés. Cependant, dans le but d'affronter de façon plus complète les problèmes spécifiques posés par la transmission culturelle, des incursions sont parfois effectuées dans le domaine littéraire. En début d'année, chaque étudiant a été invité à fournir à l'enseignant quelques textes qu'il estime propres à être traduits pour un lectorat français; il a indiqué d'une phrase le domaine concerné et l'idée générale. L'enseignant dispose ainsi d'un petit stock d'articles récents, dont chacun peut faire le support d'une séquence d'entraînement comme celle que nous allons évoquer dans ce qui suit.

\section{A. Préparation}

Avant même d'avoir pris connaissance du document de travail, le groupe est interrogé sur le thème concerné, par exemple: la condition de la femme en Corée...». Les étudiants ont le temps de mener une petite réflexion à plusieurs voix, soutenue par les questions incitatives de l'enseignant, leur permettant de rassembler leurs connaissances et leurs idées sur le sujet. L'enseignant suggère ensuite une comparaison avec la France et avec d'autres sociétés européennes connues de certains étudiants, puis termine par un petit résumé de l'évolution du droit des femmes dans la France du $\mathrm{xx}^{\mathrm{e}}$ siècle.

Ce préambule n'est pas seulement le rappel d'un chapitre du cours de civilisation. Il est une première préparation au travail de traduction, servant, d'une part, à revoir, préciser et déjà étendre les éléments de lexique qui risquent fort d'être utilisés, d'autre part, à prendre la mesure de l'écart existant entre les deux cultures, c'est-àdire entre l'implicite culturel du lecteur visé par le texte original et celui du lecteur visé par le texte traduit. Le traducteur se dispose ainsi à prendre en compte ce dernier en lui faisant comprendre, chaque fois que ce sera nécessaire, les représentations qui sont évidentes pour le premier. 
Le texte est alors communiqué aux étudiants. Quelques questions amènent une détermination des conditions dans lesquelles il a été lu par son public cible. C'était le 4 septembre 2001 ; le groupe se rappelle qu'à ce moment-là, on commençait à parler beaucoup de la condition des femmes, des femmes au travail. Un ministère spécifique venait d'être créé, etc. L'article est extrait d'un hebdomadaire économique (Dot 21) que l'on situe au centre-gauche. Selon les étudiants, il s'agit plutôt d'une sensibilisation destinée au grand public: on pose par hypothèse que le texte sera traduit pour un lectorat français de niveau équivalent. Le style est courant, parfois familier, permettant d'établir une complicité facile avec le lecteur : tonalité qu'il faudra respecter, car elle fait partie, de plein droit, du «sens» du texte.

Cette étape correspond à ce qu'il est convenu d'appeler « détermination du contexte pragmatique» ou de «l'entour pragmatique», englobant tout ce qui fait partie de la situation de communication. Il s'agit de décrire, en fonction de la date et du support, les connaissances et les attentes que le journaliste sait présentes chez son lecteur et le type d'impact que le lecteur sait que ce journal cherche d'ordinaire à l'avoir sur son lectorat. Tout cela, en grande partie ignoré de celui qui sera le lecteur second, intervient dans le sens du texte et n'est cependant pas écrit. C'est donc encore un implicite dont le traducteur devra tenir compte.

Dans l'entraînement en régime classique, cette première phase de travail, qui est une prise de contact avec les différents paramètres du sens, est un préalable nécessaire. Mais, dans les conditions où nous nous trouvons, l'éloignement culturel est tel que tout ce pan du sens risquerait d'échapper totalement au lecteur second: dès lors, ce préalable devient incontournable.

\section{B. Résumé}

Après une seconde lecture plus détaillée, mission est donnée de repérer les jalons importants de l'itinéraire. Dans cette étape, qui est le premier travail portant réellement sur le texte, il ne s'agit pas encore de le traduire, mais de prendre conscience de son déroulement. Cette phase de résumé permet de parvenir à une vision globale de l'objet à traduire: ce que le texte veut vraiment dire et les moyens qu'il se donne pour le faire passer. "Rédigé dans un but bien défini, ce texte doit accomplir une action, provoquer une réaction, combler une attente ${ }^{15}$.» Au terme du résumé, qui est aussi une analyse, le texte apparaît ainsi comme une unité communicative ayant sa fonction propre, avec son idée-force, ses caractéristiques illocutoires et perlocutoires.

Une fois posé le sens de l'ensemble, la même technique peut être appliquée aux parties et aux sous-parties du texte: le résumé se rapproche ainsi de plus en plus d'une paraphrase. Pratiquée dans une langue autre que celle du texte, elle contraint les étudiants à oublier très vite les mots utilisés dans celui-ci. Ils comprennent ainsi que le sens est quelque chose qui n'est pas attaché aux mots servant à l'exprimer; il peut en être dégagé, il peut être reproduit en utilisant d'autres mots. C'est l'intégralité du sens qui doit être décrite de cette manière, car l'enseignant ne possède pas d'autre moyen d'accéder à celui-ci. Ce travail illustre de façon éloquente le moment appelé, dans la Théorie Interprétative, "phase de déverbalisation ${ }^{16}$ ». Elle aboutit à l'établissement, libéré de la langue de départ, de ce qui sera l'invariant de la traduction. Ce travail, effectué dans la langue d'arrivée, commence, de façon encore spontanée et peu contraignante, à permettre cette reformulation, dite aussi "reverbalisation ${ }^{17}$ ", 
qui, un peu plus tard, deviendra la traduction proprement dite. Ainsi construite à partir du sens et non à partir des formulations de la langue originale, celle-ci aura toutes les chances d'échapper aux défauts classiques du «transcodage».

\section{Traduction à vue}

Un segment de texte de quelques lignes est alors confié à l'un des étudiants qui est prié de le traduire, au rythme qui lui convient et sans être interrompu. Chaque segment doit être suffisamment court pour que les auditeurs l'aient entièrement présent à l'esprit le temps de la traduction et de l'échange qui suivra, mais suffisamment long pour permettre d'observer l'enchaînement de plusieurs idées ou informations. L'enseignant prend intégralement en note ce premier jet, afin de répéter la traduction donnée et de susciter la critique constructive du traducteur et du public. Voici, à titre d'exemple, le premier segment du texte:

Il y a quelques jours, j'ai rencontré un ami; il vient d'avoir un premier enfant; il avait l'air un peu fatigué. Il m'a dit qu'il ne faut pas faire toujours confiance à ce que les adultes disent. Normalement, on dit qu'un enfant, il grandit tout seul, mais mon ami, il a remarqué que ce n'est pas toujours vrai. S'il avait eu le moindre doute de ce dicton, il n'aurait pas eu cet enfant.

On discute certains points :

- Selon le traducteur, il y a une tournure typiquement coréenne pour exprimer l'idée toute faite sur les enfants. Il faut donc trouver une expression française qui fasse cliché; peut-être: «un enfant, ça pousse tout seul...»

_ $\quad$ «Les adultes» : cela signifie-t-il que le jeune père n'est pas un adulte? On propose : «les anciens », «les aînés», «les ancêtres»; l'enseignant précise les différences entre ces mots.

- Le temps des verbes: le traducteur est prié de justifier son choix du passé composé. On décide de le conserver.

- «le moindre doute $\underline{\text { de }}$ ce dicton»; correction: «le moindre doute concernant ce dicton».

- Quelqu'un dit que la fin est un peu résumée. Plusieurs donnent leur avis; on aboutit à: «Il aurait réfléchi un peu plus avant d'avoir un enfant.»

La traduction à vue fait partie, en tant que telle, des compétences attendues d'un traducteur professionnel. Le travail «à chaud» est donc, de ce point de vue, amplement justifié. Mais il comporte surtout des bénéfices didactiques considérables. Le premier d'entre eux provient de l'urgence. Dans cette traduction improvisée et en groupe, l'étudiant responsable n'a pas le temps de se laisser fasciner par les mots du texte. Une fois le sens compris, il faut le faire passer; l'auditoire attend et cette urgence mobilise au maximum les ressources expressives. Le second bénéfice est produit par le caractère collectif du travail, qui nous amène à utiliser la notion de «négociation ». Le sens du texte original est l'objet d'une négociation entre les étudiants, discussion menée en français et qui fait souvent surgir de bonnes formulations, à l'insu des participants, mais remarquées par l'enseignant. Lorsqu'il semble vraiment y avoir désaccord, ce dernier suggère une brève « mise à plat» en coréen. Quant à la traduction, elle est l'objet d'une négociation entre le groupe et l'enseignant, le groupe proposant les formulations françaises qui lui paraissent adaptées, l'enseignant explicitant ce que le lecteur français comprendra sous la formulation qu'on lui propose et déclenchant ainsi les rectifications nécessaires: le texte français s'élabore au cours de toute une série d'allers et retours entre le sens et sa reverbalisation. Les corrections au niveau du 
français - qui sont faites brièvement, car le but de l'entraînement n'est pas l'apprentissage de la langue - amènent souvent, elles aussi, un retour vers le sens.

Dans cette phase, le rôle du francophone est, volontairement ou involontairement, celui d'un candide qui veut comprendre, qui, parfois, comprend de travers et dont les questions stimulent la créativité linguistique: pour lui, on cherche d'autres mots qui feront mieux passer le sens. Le travail porte aussi sur les arrière-plans culturels, l'explicitation d'une donnée implicite étant faite spontanément par le groupe ou amenée par une question du francophone. Durant toute cette élucidation, l'enseignant doit faire preuve d'une extrême vigilance pour être prêt à réagir au moindre indice signalant un implicite non clarifié qui menacerait la lisibilité de la future traduction.

Ce moment bouillonnant et créatif fait apparaître très clairement le bénéfice didactique de la situation. Au niveau linguistique, la contrainte de dissociation est d'emblée portée à son maximum, compréhension et réexpression étant incarnées par des personnes physiques différentes. Mais c'est au niveau culturel que ce bénéfice est le plus grand, l'altérité culturelle étant ici la base même de la relation pédagogique. La communication n'est pas donnée, elle est à construire; la traduction s'élabore comme une négociation in presentia entre le groupe et l'enseignant, comme elle sera plus tard une négociation in absentia entre le traducteur et son lecteur.

\section{Traduction écrite}

Entre cette séance et la suivante, les étudiants font les recherches suggérées et d'autres - c'est la phase de recherche documentaire - et rédigent une première traduction écrite; la deuxième séance est consacrée à la lecture et au commentaire de celle-ci. Comparé au premier jet, on constate bien sûr des améliorations: les phrases sont plus liées, plus longues, avec des mots de liaison et des mises en relief. Des éléments omis ont été rétablis. Un enrichissement lexical a eu lieu, avec l'apparition de mots nouveaux, dont certains sont très à leur place dans le contexte. Enfin, la recherche documentaire se manifeste par une meilleure précision au niveau terminologique. Un travail de maturation indispensable a donc eu lieu. Mais ces améliorations ont leur contrepartie: les formulations sont souvent moins authentiques que dans l'improvisation, avec des maladresses et des incorrections qui n'étaient pas dans la première phase. Les traductions sont quelquefois trop explicitées, avec un excès de liaisons ou des liaisons mal choisies. Ces déficiences sont la trace d'un travail solitaire sur le texte, au cours duquel le traducteur a été de nouveau exposé à la fascination de la langue source, risquant de retomber dans les erreurs dues au transcodage. La reprise collective a pour but d'en faire prendre conscience.

Au cours de cette deuxième séance, on discute également de problèmes mettant en jeu des aspects plus spécifiquement culturels. Quel nom convient-il de donner à certaines réalités propres au pays d'origine, comme ici le nom du ministère? Faut-il garder le calque de l'expression coréenne ( «le Ministère de la Femme») ou adopter une expression plus proche de ce que connaît le lecteur français ( «ministère de la Promotion de la femme», "des Droits de la femme»), mais qui risque d'être une traduction «ethnocentrique»? Comment formuler le titre? Les titres proposés par les étudiants coréens sont souvent longs et explicites, alors que les habitudes françaises privilégient des titres-choc, qui interpellent plus qu'ils ne répondent. Il peut y avoir lieu aussi d'améliorer la cohésion entre phrases et la cohérence au niveau de l'ensemble 
du texte: supprimer des répétitions, procéder à des resserrements, éventuellement regrouper des paragraphes. Ces techniques ont pour but de rapprocher le texte traduit des habitudes et des attentes du lecteur français, donc d'améliorer la transmission du message. Le nouveau lecteur ne doit pas être gêné par une formulation qui lui donnerait une impression d'étrangeté et ferait naître un sentiment d' « exotisme» face à la culture d'origine.

Entre la deuxième et la troisième séance, les traductions écrites ont été lues et corrigées par l'enseignant. La troisième séance est l'occasion de faire les dernière mises au point stylistiques et de publier les bonnes solutions trouvées. Une «traduction finale", synthèse écrite du travail collectif, est quelquefois proposée, à la différence du cours de traduction classique: il est clair que, dans les conditions de travail décrites, la traduction finale n'est pas reçue comme un "corrigé», mais comme le résultat d'une coopération. Lorsqu'il s'avère qu'une compréhension non réductrice de l'autre - ou de soi par l'autre - a été possible, sa lecture collective est alors un moment de bonheur.

\section{La question de l'évaluation}

Comme l'enseignement en «régime spécial» débouche sur la délivrance d'un certificat, il faut qu'intervienne une évaluation, c'est-à-dire une procédure de mesure de la compétence acquise. Dans l'université française, le passage obligé de l'évaluation est l'examen ou le «partiel», avec leurs règles strictes. Ces conditions sont très différentes de celles de l'entraînement précédemment décrit, où l'on a vu l'importance du travail collectif, et très différentes aussi de la pratique professionnelle. Le moment de l'évaluation risque donc d'être problématique. Il l'est plus encore si l'on considère que l'enseignant a besoin de la présence des étudiants pour la compréhension du sens. Nous allons montrer par un exemple les procédures par lesquelles ces problèmes peuvent être résolus.

Le texte d'examen, comme les textes d'entraînement, est choisi parmi les textes proposés en début d'année ou parmi les textes traités l'année précédente. Les critères de choix se résument à trois: la longueur (le texte, si possible intégral, doit être gérable en une heure et demie), le thème traité (il ne doit pas mettre en jeu de terminologie spécifique ni de connaissances spécialisées) et le type de texte (un texte argumentatif permet, plus efficacement qu'un autre, de juger la maîtrise de l'expression française). Dans l'exemple étudié, l'article présente l'annonce, par le ministère de l'Éducation sud-coréen, de la décision d'accorder à un certain nombre de lycées une large part d'autonomie pédagogique et financière (éditorial du quotidien Chungang Ilbo, août 2001).

La méthode que nous présentons consiste en trois lectures successives de l'ensemble des copies, chaque lecture donnant lieu à une observation différente et à une évaluation chiffrée distincte.

\section{A. Evaluation «verticale»}

La première lecture doit permettre d'apprécier chaque texte traduit en tant que texte français autonome, indépendamment de tout rapport à un texte original et à d'autres traductions du même article. Cette partie de l'évaluation est la première dans l'ordre 
chronologique, car, avant d'être un professionnel de la traduction, le correcteur est d'abord un lecteur. Chaque traduction est lue intégralement du début à la fin, d'où notre expression, faute d'un terme plus conceptuel, d'évaluation «verticale». L'évaluation «verticale» consiste à apprécier la lisibilité du texte traduit: le lecteur français doit pouvoir comprendre la pertinence de l'information qu'on veut lui transmettre, saisir le bien-fondé de l'argumentation qu'on lui demande de suivre, ressentir les mouvements émotionnels qui assurent l'efficacité du message. De plus, il doit pouvoir le faire sans fournir un effort particulier d'interprétation : la bonne réception est au prix d'un accès au sens qui soit facile et agréable. Il va de soi que la lisibilité de la traduction d'un article de presse ne suppose pas des qualités de style qui seraient mesurées à l'aune de l'écriture littéraire.

Lorsque la lisibilité n'est pas assurée dans de bonnes conditions, il peut s'agir d'une traduction qui «colle» de trop près aux structures de la langue de départ: dans ce cas, l'étudiant a insuffisamment assimilé la technique de déverbalisation exposée précédemment. Il peut s'agir aussi, malgré le bon niveau de sélection des étudiants, d'une maîtrise insuffisante du français écrit, qui va obérer la bonne réception du sens par le futur lecteur; pertinence du lexique employé, exactitude morphologique, correction des syntagmes, de la syntaxe et aisance générale du style doivent donc être observées avec soin.

Dans ce domaine, le problème le plus résistant pour les étudiants et leur enseignant est celui des «obscurités ». Il y a obscurité lorsqu'une phrase n'est pas interprétable selon le jeu normal du mécanisme de lecture, mais uniquement après un travail de réflexion qui interrompt celui-ci, ou, plus gravement, lorsqu'elle n'est pas compréhensible $d u$ tout. Les obscurités sont l'obstacle principal à la transmission du sens. Elles peuvent souvent être imputées à l'un ou l'autre des types d'incorrection précédemment évoqués, ou, plus largement, à des manquements à l'usage: mauvaise organisation de la phrase, concernant, par exemple, la place des compléments circonstanciels ( "Les lycées indépendants procéderont à la sélection des élèves auprès des collégiens en troisième année, en fonction du résultat obtenu au collège, de compétence et d'aptitude à partir de décembre de cette année.»), choix d'un mot de liaison inadéquat (à cet égard, la relation concessive s'avère d'un maniement particulièrement délicat), gestion des répétitions, qui constituent un sujet difficile pour les apprenants de langue maternelle asiatique. Au-delà de leur analyse strictement linguistique, elles font souvent apparaître des zones très secrètes de l'implicite culturel. En situation d'évaluation, elles n'en sont pas moins comptabilisées comme des «erreurs».

\section{B. Évaluation «horizontale»}

Le but de la deuxième lecture est d'apprécier la conformité de chaque traduction au sens du texte original. N'ayant pas lui-même accès à la langue de départ, l'enseignant doit admettre, à titre provisoire, que le sens d'un passage est celui qui a été identifié par la majorité des traducteurs; il s'agit d'une hypothèse de travail qui, si elle se révèle juste dans la grande majorité des cas, devra cependant, pour chacun d'eux, être vérifiée. S'agissant d'une traduction en $\mathrm{B}$, on ne rencontrera pas de traduction où la compréhension du sens serait gravement fautive; les seules déviations que l'on détecte sont minimes, et souvent réductibles à des maladresses d'expression qui provoquent un «effet» d'erreur. Ce contrôle d'exactitude doit cependant être effectué. 
Pour cela, le texte est divisé en segments, définis comme au cours de l'entraînement. L'évaluation se fait, pour chaque segment, au moyen d'un examen comparatif du contenu de sens de toutes les traductions: d'où notre expression d'évaluation «horizontale». À titre d'exemple, voici deux formulations proposées pour le début du texte:

Le ministère coréen de l'éducation et des ressources humaines a décidé de mettre en place à titre expérimental des lycées indépendants à partir de l'an prochain, qui auront une liberté totale de sélectionner leurs élèves et de fixer les frais d'inscription et les cursus.

Le ministère de l'Education et des Ressources humaines a décidé d'expérimenter, à partir de l'année prochaine, un nouveau type de lycées privés: ceux-ci bénéficieront d'une autonomie dans la sélection de leurs élèves, la fixation des frais scolaires et la gestion des programmes pédagogiques.

La but est de vérifier l'invariance du contenu derrière des formulations qui peuvent être très différentes, en fractionnant le sens global du segment en un certain nombre d'unités de sens. Pour ne pas entrer dans un débat théorique qui serait complexe, la notion d'unité de sens doit être comprise ici de façon opérationnelle: on la définira comme «le plus petit élément qui permette l'établissement d'équivalence en traduction $^{18}$ ». Dans le segment cité, l'unité de sens que nous désignerons par /à titre d'essai/ peut être reconnue derrière les différentes formulations proposées: «gestion pilote», «dans les lycées pilotes», «à titre expérimental», «à des fins d'essai », « expérimenter». Cette méthode permet d'identifier avec une relative certitude les passages «déviants», définis comme ceux où une unité de sens serait omise, ajoutée ou modifiée.

On entrevoit déjà pour cette méthode quelques difficultés d'application. Elle suppose d'abord de la part du correcteur l'effort de faire abstraction des qualités ou des défaillances de l'expression française, déjà évaluées lors de la correction «verticale», et de se concentrer sur le seul contenu de sens. De plus, contrairement à ce qui advient généralement dans le passage entre langues européennes, les traductions ne reproduisent pas un découpage du sens déjà effectué par la succession des phrases du texte original, mais restructurent fortement et diversement son organisation: pour une même unité de sens, la formulation équivalente peut donc figurer à des places très différentes à l'intérieur du segment traduit, voire dans le segment précédent ou le segment suivant. Notons aussi que le «coefficient de foisonnement» peut être très différent d'un traducteur à l'autre. Cette constatation impose au correcteur une grande prudence dans l'identification d'un «ajout», qui peut être simplement le résultat d'une stratégie d'explicitation; d'une "omission», qui peut être une traduction simple là où les autres ont pratiqué une explicitation. Enfin, pour certaines notions, le français impose une interprétation tranchée que le texte coréen ne permet pas; dans ce cas, il est malaisé de reconnaître dans les différentes traductions un sens qui serait clairement «majoritaire».

Pour cette partie de l'évaluation, une vérification en séance collective est indispensable. Chaque «erreur» identifiée par l'enseignant doit être confirmée comme telle par le groupe: une concertation a donc lieu et l'identification d'une «erreur» est arrêtée seulement après accord de l'étudiant concerné et des autres.

L'expérience montre que, moyennant ces précautions, et à condition de disposer d'un nombre suffisant de traductions, la correction «horizontale» est, certes, une méthode laborieuse, mais qu'elle permet de vérifier avec une assez grande fiabilité la fidélité au sens. 


\section{Transmission des éléments culturels}

La dernière lecture ne concerne pas l'ensemble du texte, mais uniquement les unités qui, dans la culture d'origine du texte, font appel à une connaissance encyclopédique spécifique venant participer au sens général ${ }^{19}$. Or «les objets ou notions appartenant exclusivement à une culture donnée ne possèdent pas de correspondance lexicale dans la civilisation d'accueil et si on arrive à les exprimer néanmoins, on ne peut compter sur le lecteur de la traduction pour [en] connaître avec précision la nature ${ }^{20} »$. Dans l'optique de notre formation et de ses objectifs, le traitement de ces particularités culturelles revêt une importance toute particulière. Pour ces passages, la lisibilité du texte traduit suppose un travail de mise en conformité avec le bagage cognitif du nouveau lecteur: une opération d'explicitation doit être pratiquée, dans une mesure qui ne peut être déterminée que cas par cas; elle doit être aussi développée qu’il est nécessaire, aussi discrète qu'il est possible. De plus, dans une traduction en $\mathrm{B}$, la juste mesure de l'adaptation culturelle au lecteur est plus difficile à déterminer que dans une traduction en $\mathrm{A}$ : pour mesurer avec précision les lacunes qu'il lui convient de combler, le traducteur a besoin d'une connaissance très vivante de la culture de son lecteur.

Deux exemples figurant dans l'article choisi permettront d'illustrer la difficulté variable de cette opération.

Dans de nombreux cas, une explicitation ponctuelle judicieuse peut suffire. Pour le lecteur français moyennement informé, l'information selon laquelle «à Kangnam, on voit apparaître des cours privés préparant les élèves à être admis dans les nouveaux lycées» ne saurait convenir; son maintien sous cette forme constituerait une erreur de traduction. Une explicitation comme «le quartier de Kangnam de Séoul» est utile, mais insuffisante, car elle ne fait pas apparaître la pertinence du mot dans le passage. En revanche, la traduction «les quartiers riches comme Kangnam, dans le sud de Séoul» est très satisfaisante.

Il arrive aussi que la compréhension d'un texte entier repose sur une notion spécifique à sa culture d'origine. Dans l'exemple évoqué, le lecteur français a ressenti une gêne face à plusieurs passages du texte. L'analyse de cette incompréhension n'a pu se faire qu'en séance collective, grâce à un travail portant sur la comparaison des deux systèmes scolaires: il est alors apparu que l'expression "lycée privé», spontanément utilisée par la majorité des étudiants, évoquait chez le lecteur français une notion qui ne correspondait pas à la réalité coréenne en cause. Il convenait de parler de «lycées fondés par le fait d'une initiative privée», en ajoutant à un autre endroit du texte la mention explicite de «la part de financement assurée par les fondations qui sont à l'origine de ces lycées ». Dès lors, la pertinence de l'ensemble du texte est devenue évidente.

$\mathrm{Au}$ terme des trois lectures, une synthèse doit permettre d'intégrer en une seule note les différents éléments analysés ci-dessus, tout en les pondérant selon leur importance. Dans le mode d'évaluation présenté, l'appréciation du texte en langue cible et la technique de transmission des éléments culturels sont les paramètres les plus fortement pris en compte. Dans cette mesure, ce mode d'évaluation est particulièrement adapté aux principes de la formation des traducteurs de langue périphérique en langue $\mathrm{B}$. 


\section{Conclusion}

Le sort des langues périphériques et des cultures dont elles sont solidaires est entre les mains des jeunes traducteurs natifs, qui seront amenés à travailler vers le $\mathrm{B}$, et des instituts prêts à assurer leur formation. L'expérience décrite ici et les réflexions qu'elle a suscitées montrent qu'il est possible de concevoir une didactique de la traduction qui soit une didactique générale tout en s'adaptant aux contraintes de cette formation.

L'entraînement en B se prête à toute une maïeutique permettant aux étudiants de découvrir par eux-mêmes les exigences de leur travail, à une progression lente qui permettra de «faire basculer dans l'automatisme ce qui, une première fois, a été le fruit d'un effort ${ }^{21} »$. Il peut donc, tout autant que l'entraînement en $\mathrm{A}$, faire acquérir cette méthodologie qui, on le sait, est la même pour tous les couples de langues. Quant aux procédures de formation et d'évaluation auxquelles est contraint de recourir un enseignant n'ayant pas accès à la langue maternelle des étudiants, elles sont, certes, plus difficiles à mettre en œuvre que les procédures de type classique, mais elles valorisent de la même façon les qualités, comme la prise en compte du lecteur, qui font la différence entre la formation professionnelle et une formation linguistique de type universitaire. Enfin, cette formation habitue les futurs traducteurs à recourir au regard d'un natif - qui, plus tard, sera le réviseur - pour valider définitivement une traduction vers le $\mathrm{B}$.

Au-delà de la didactique, il reste à dire ce que cette expérience apporte au domaine de la traductologie. Concernant la traduction en B, nous n'avons, d'un point de vue théorique, aucune raison de contester les conclusions de travaux effectués antérieurement: dans le cas particulier qui nous a retenu, il reste également incontestable que «à qualité égale de traducteur, aucune traduction en $\mathrm{B}$ ne peut rivaliser avec une traduction en langue A. ${ }^{22}$ » Mais, comme l'auteur cité le fait également remarquer, ces réserves ne sont pas décisives face à la réalité et à l'importance des enjeux ${ }^{23}$. De fait, une traduction effectuée selon les processus décrits précédemment a toutes les chances d'être une traduction satisfaisante, dans la mesure où elle s'efforce de remplir les critères d'équivalence ${ }^{24}$ et où - l'expérience l'a prouvé - elle y parvient. Concernant les mécanismes de l'opération traduisante, la pratique décrite ici permet une observation à la loupe du travail du traducteur, que ce soit le rééquilibrage de l'implicite et de l'explicite ${ }^{25}$ ou l'articulation des phases de déverbalisation et reverbalisation; elle constitue ainsi une remarquable illustration de l'analyse proposée par la Théorie Interprétative. Enfin, cette didactique met particulièrement en évidence les véritables enjeux de la traduction: la relation pédagogique montre à l'évidence, et ce, d'autant plus fortement que les cultures mises en contact sont plus lointaines, que «la traduction n'est pas une simple médiation: c'est un processus où se joue tout notre rapport avec l'Autre ${ }^{26} »$.

Dans cette perspective, il semble acquis que l'on peut compter, pour relever les défis de la défense du pluralisme linguistique et culturel à l'ère de la mondialisation, sur des professionnels qui seront en mesure de porter le flambeau de leur culture tout en mettant sur le marché des traductions de qualité: tels sont les professionnels formés selon les principes de la Théorie Interprétative, dans le cadre de ce «régime» très «spécial», qui, loin d'être un pis-aller, est une spécificité dont l'ÉSIT a le droit d'être fière. 


\section{NOTES}

1. École Supérieure d'Interprètes et de Traducteurs, Paris III.

2. L.-J. Calvet, 1999, 75-83, pour l'ensemble des passages cités.

3. Ce sont celles que nous avons dites «de petite diffusion» et que l'on qualifie parfois de «petites» langues ou «langues exotiques».

4. L'auteur prévient que, dans le détail, «les choses peuvent être plus compliquées, en particulier parce que $[\ldots]$ une même langue peut être présente dans deux constellations différentes, être ici centrale et ailleurs périphérique».

5. Ce n'est pas une règle absolue: la France, par exemple, compte quelques maisons d'édition extrêmement attentives aux "domaines étrangers", aussi lointains soient-ils.

6. «Détours de Babel», par N. Levisalles, Libération du 9 octobre 2003. Ces chiffres demanderaient bien sûrs à être précisés et complétés.

7. Pour ce qui est des langues, citons, parmi beaucoup d'autres, l'estimation de D. Crystal (Prospect, cité par Courrier International, hors série 2003), selon laquelle la moitié des langues du monde seraient appelées à disparaître au cours de ce siècle. Quant aux cultures, les ethnologues sont témoins, sous toutes les latitudes, d'une dénaturation puis d'une disparition rapide des cultures singulières. J.-P. Warnier $(1999,77)$ parle à ce sujet d'une "apocalypse des traditions».

8. Même si l'évocation, qui pourrait être faite par extrapolation, d'une uniformisation culturelle généralisée ne semble pas résister à l'examen. Voir à ce sujet J.-P.Warnier (1999, 91 et suite)

9. Dans le sens où l'on parle de «commerce équitable». Nous empruntons ce rapprochement à C. Touitou-Benitah, 1998, 367.

10. C'est l'option prise par la Communauté européenne; on en connaît les coûts, mais aussi les enjeux.

11. L'autre option étant l'organisation de stages de formation dans leur pays d'origine: voir à ce sujet Déjean le Féal, 1987.

12. Il reste que le régime «classique» constitue la formation majoritairement dispensée à l'ÉSIT, avec environ 250 étudiants, répartis sur 3 ans; le «régime spécial » concerne, selon les années, entre 30 et 40 étudiants.

13. Pour le domaine de l'interprétation, une didactique répondant à des exigences similaires a été présentée par K. Déjean Le Féal (1990).

14. Cette étude de cas a fait l'objet d'une publication plus détaillée dans la revue franco-coréenne FORUM.

15. F. Herbulot, 1990, 269.

16. Voir M. Lederer, 1994, 22 et suite.

17. Voir M. Lederer, 1994, 43 et suite.

18. M. Lederer, 1994, 27

19. Cette définition des «éléments culturels» est naturellement restrictive, toutes les représentations véhiculées par le texte et sa manière de les transmettre étant également d'ordre culturel. On a cependant intérêt, d'un point de vue didactique, à isoler les marqueurs culturels les plus reconnaissables.

20. M. Lederer, 1994, 122.

21. D. Seleskovitch, cité par M. Cormier, 1990, 173.

22. M. Lederer, 1994, 149.

23. Voir aussi A. F. Rydning, dont la thèse traite spécifiquement de la traduction en langue B.

24. Selon les paramètres de W. Koller (1979), par exemple.

25. Voir M. Lederer, 1994, 58-62.

26. A. Berman, 1984, 287.

\section{RÉFÉRENCES}

Berman, A. (1984): L'épreuve de l'étranger, Paris, Gallimard.

Calvet, L.-J. (1999): Pour une écologie des langues du monde, Paris, Plon.

Cormier, M. (1990): «Proposition d'une typologie pour l'enseignement de la traduction technique», Études traductologiques en hommage à D. Seleskovitch, Minard, Paris.

DÉJEAN LE FÉAL, K. (1990) : «La formation méthodologique d'interprètes de "langues exotiques" ", Études traductologiques en hommage à D. Seleskovitch, Paris, Minard.

DéjEAN LE FÉAL, K. (1987): «La traduction à l'approche de l’an $2000 »$, META 32-2. 
Herbulot, F. (1990) : «Le traducteur déchiré», Études traductologiques en hommage à D. Seleskovitch, Paris, Minard.

Herbulot, F. (2004): «Le régime spécial ou l'enseignement de la traduction en français à partir d'une langue 'exotique' que l'enseignant ne connait pas», La Théorie Interprétative de la traduction: regards croisés (à paraître), Paris, Minard.

Koller, W. (1979) : Einführung in die Übersetzungswissenschaft, Quelle und Meyer, Heidelberg.

Lederer, M. (1994): La traduction aujourd'hui: le modèle interprétatif, Paris, Hachette.

Roux-Faucard, G. (2004): «À propos de la traduction du coréen à l'ÉSIT», FORUM, Paris, Presses de la Sorbonne Nouvelle et Séoul, KSCI.

Rydning, A. F. (1991): Qu'est-ce qu'une traduction acceptable en B?, thèse, Oslo.

Seleskovitch, D. (1986) : «L'enseignement de l'interprétation», Interpréter pour traduire, Paris, Didier Erudition.

Touitou-Benitah, C. (1998): «Le modèle de la traduction en Europe», Europe et traduction, Aras, Artois Presses Université.

Warnier, J.-P. (1999): La mondialisation de la culture, Paris, La Découverte. 